\title{
Morphology and segregation in two-component diffusion-limited aggregation
}

\author{
Takashi Nagatani \\ College of Engineering, Shizuoka University, Hamamatsu 432, Japan \\ and Department de Quimica-Fisica, Universitat de Barcelona, Diagonal 647, E-08028 Barcelona, Spain \\ Francesc Sagués \\ Department de Quimica-Fisica, Universitat de Barcelona, Diagonal 647, E-08028 Barcelona, Spain
}

(Received 30 May 1991)

\begin{abstract}
A diffusion-limited-aggregation (DLA) model with two components ( $A$ and $B$ species) is presented to investigate the structure of the composite deposits. The sticking probability $P_{A B}\left(=P_{B A}\right)$ between the different species is introduced into the original DLA model. By using computer simulation it is shown that various patterns are produced with varying the sticking probabilities $P_{A B}\left(=P_{B A}\right)$ and $P_{A A}$ ( $\left.=P_{B B}\right)$, where $P_{A A}\left(=P_{B B}\right)$ is the sticking probability between the same species. Segregated patterns can be analyzed under the condition $P_{A B}<P_{A A}$, assumed throughout the paper. With decreasing sticking probability $P_{A B}$, a clustering of the same species occurs. With sufficiently small values of both sticking probabilities $P_{A B}$ and $P_{A A}$, the deposit becomes dense and the segregated patterns of the composite deposit show a striped structure. The effect of the concentration on the pattern morphology is also shown.
\end{abstract}

PACS number(s): 68.70. $+\mathrm{w}, 05.70 . \mathrm{Jk}, 05.40 .+\mathrm{j}$

\section{INTRODUCTION}

Recently there has been increasing interest in fractalgrowth phenomena under nonequilibrium conditions [1-9]. Laplacian growth phenomena in pattern formation have attracted considerable attention [10]. The Laplacian growth model is consistent with the diffusionlimited-aggregation (DLA) model $[11,12]$. The DLA model presents a prototype of the pattern formation of diffusive systems including the electrochemical deposition, crystal growth, viscous fingering, dielectric breakdown, chemical dissolution [13], and bacterial colony [14]. A variety of computer simulations have been carried out to investigate the relationships between the cluster geometry and growth mechanisms. The structure of the aggregates strongly depends on the dynamics of the growth process. The morphological changes between the DLA fractal and the nonfractals have been found by computational and experimental methods [15-20]. The morphological changes have also been analyzed by the real-space renormalization-group method [21-24].

Up to now, most of the studies on pattern-formation phenomena in this context refer to a single-component DLA. However, very few investigations for the DLA with two components have been performed. It will be interesting to study the morphological changes in the DLA with two components. In the previous paper [25] we studied the morphological change in the DLA with two immiscible components by using a computer simulation and a real-space renormalization-group method. We restricted ourselves to the limiting case where a connection between $A$ and $B$ species is inhibited. The $A$ species sticks only on the surface consisting of the $A$ species and the $B$ species sticks only on the surface consisting of the $B$ species. In the limiting case, a segregation occurs where the DLA pattern is constructed by a mixture of the trees consisting of only $A$ species and the trees consisting of only $B$ species. We found that a morphological phase transition occurs between the DLA only with $A$ species and the DLA only with $B$ species at a critical concentration [25].

In this paper we extend the previous model to the general case in which the connection between $A$ and $B$ species is taken into account, and investigate the morphological change in the DLA with two components by using a computer simulation. We introduce the sticking probability $P_{A B}$ between the different species into the original DLA model. We show that various patterns of the composite deposits are produced with varying the sticking probabilities $P_{A B}\left(=P_{B A}\right)$ and $P_{A A}\left(=P_{B B}\right)$, where $P_{A A}$ $\left(=P_{B B}\right)$ is the sticking probability between the same species. We find that a clustering of the same species occurs with decreasing sticking probability $P_{A B}$. In the limit of a sufficiently small value of $P_{A B}$, a segregation phenomenon appears and trees only with $A$ species are separated from trees only with $B$ species. With both sufficiently small sticking probabilities $P_{A B}$ and $P_{A A}$, the overall shape of the deposit approaches that of a dense pattern and the segregated patterns of the composite deposit show a vertical laminar structure. The effect of the concentration $p$ on the pattern morphology is also shown.

The organization of the paper is as follows. In Sec. II we present the model. In Sec. III we introduce the simulation algorithm and the results of our simulations with different sticking probabilities $P_{A B}$ and $P_{A A}$ and concentration $p$ are presented. Finally, Sec. IV contains a brief summary. 


\section{MODEL}

We present the DLA model with two components ( $A$ and $B$ species). We consider the deposition process on a plate. We assume that $A$ and $B$ species diffuse independently. The concentration $C_{A}$ and $C_{B}$ of diffusive components satisfy, respectively, the distinct Laplace equations under the quasistationary approximation

$$
\begin{aligned}
\nabla^{2} C_{A} & =0, \\
\nabla^{2} C_{B} & =0 .
\end{aligned}
$$

The boundary conditions far from the plate correspond to constant concentrations $C_{A}$ and $C_{B}$. We turn now to the consideration of the boundary conditions on the surface of the deposit. We define the sticking probabilities $P_{A A}, P_{B B}, P_{A B}$, and $P_{B A} \cdot P_{A A}\left(P_{B B}\right)$ indicates the sticking probability at which $A(B)$ species sticks on the sur- face of $A(B)$ species when $A(B)$ species lands on the surface of $A(B)$ species. $P_{A B}\left(P_{B A}\right)$ indicates the sticking probability at which $A(B)$ species sticks on the surface of $B(A)$ species when $A(B)$ species lands on the surface of $B(A)$ species. We restrict ourselves to the case: $P_{A A}=P_{B B}$ and $P_{A B}=P_{B A}$. The boundary conditions are given by

$$
(1-P) \frac{\partial C_{A}}{\partial n}-P C_{A}=0 \text {. }
$$

where $P=P_{A A}$ if $A$ species lands on the surface consisting of $A$ species, $P=P_{A B}$ if $A$ species lands on the surface consisting of $B$ species, and

$$
(1-P) \frac{\partial C_{B}}{\partial n}-P C_{B}=0 \text {, }
$$
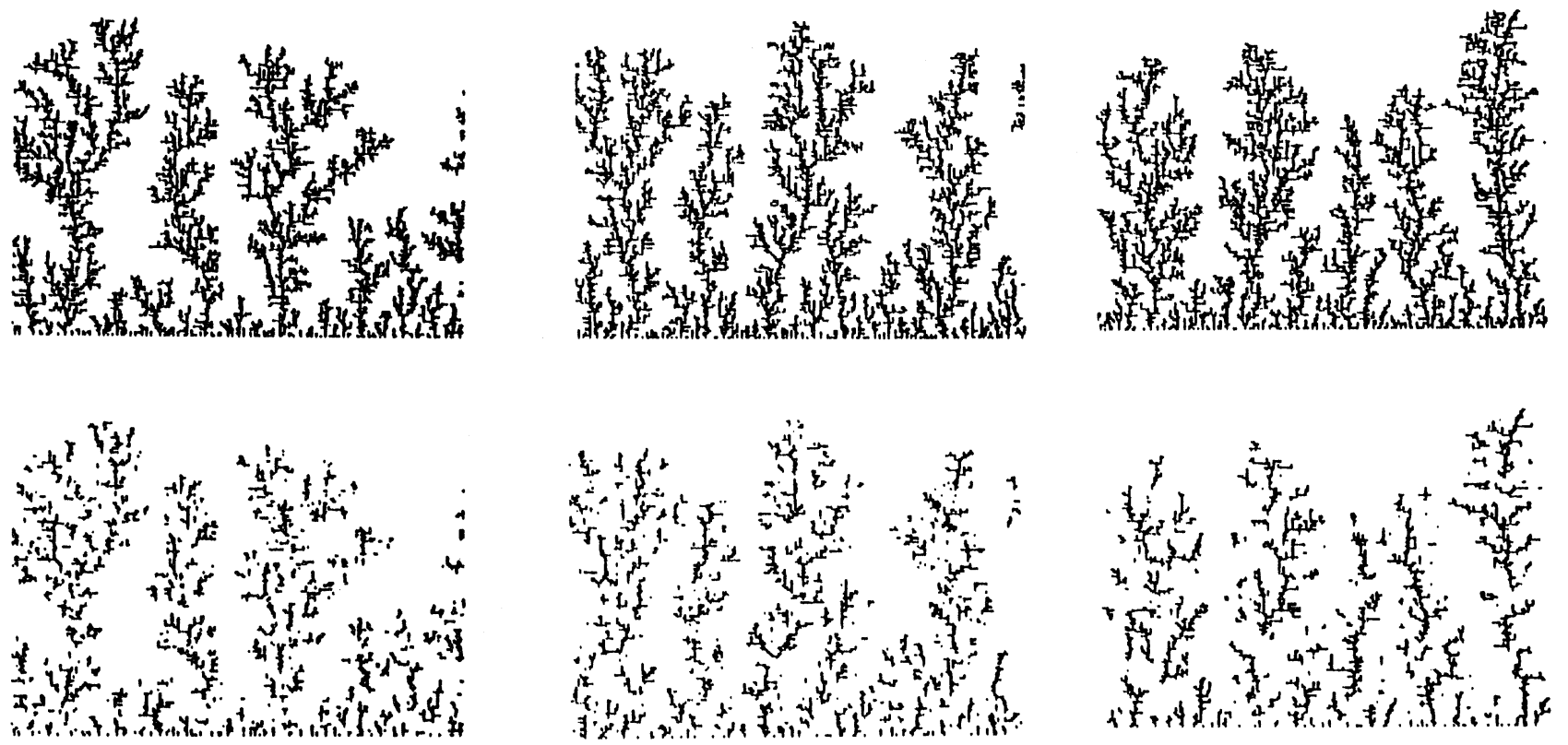

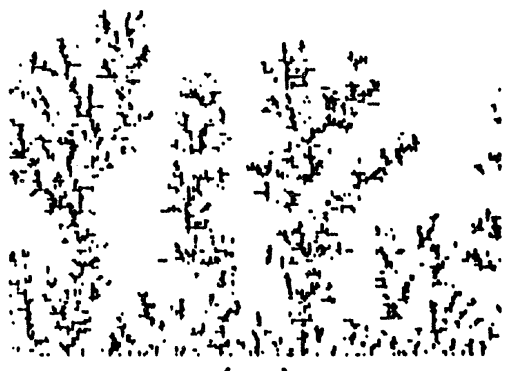

(a)
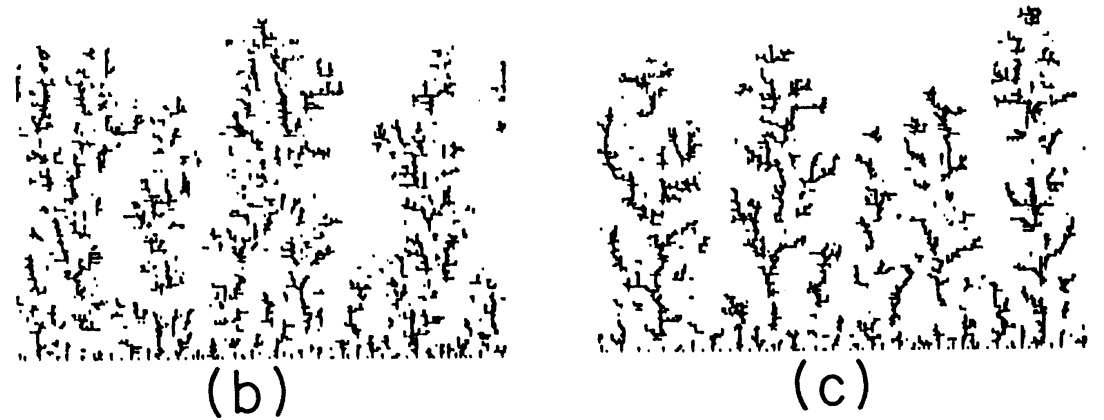

FIG. 1. Typical patterns of the composite deposits grown by varying the sticking probability $P_{A B}\left(=P_{B A}\right)$ between the different species for fixed sticking probability between the same species $P_{A A}\left(=P_{B B}\right)=1$ and composition ratio $p=0.5$. The upper, middle, and bottom patterns in this and Figs. 5-8 indicate, respectively, the composite deposits consisting of $A$ and $B$ species, only the $A$ species within the composite deposit, and only the $B$ species within the composite deposit. (a) $P_{A B}=0.05$. (b) $P_{A B}=0.03$. (c) $P_{A B}=0.01$. (d) $P_{A B}=0.005$. (e) $P_{A B}=0.001$. 

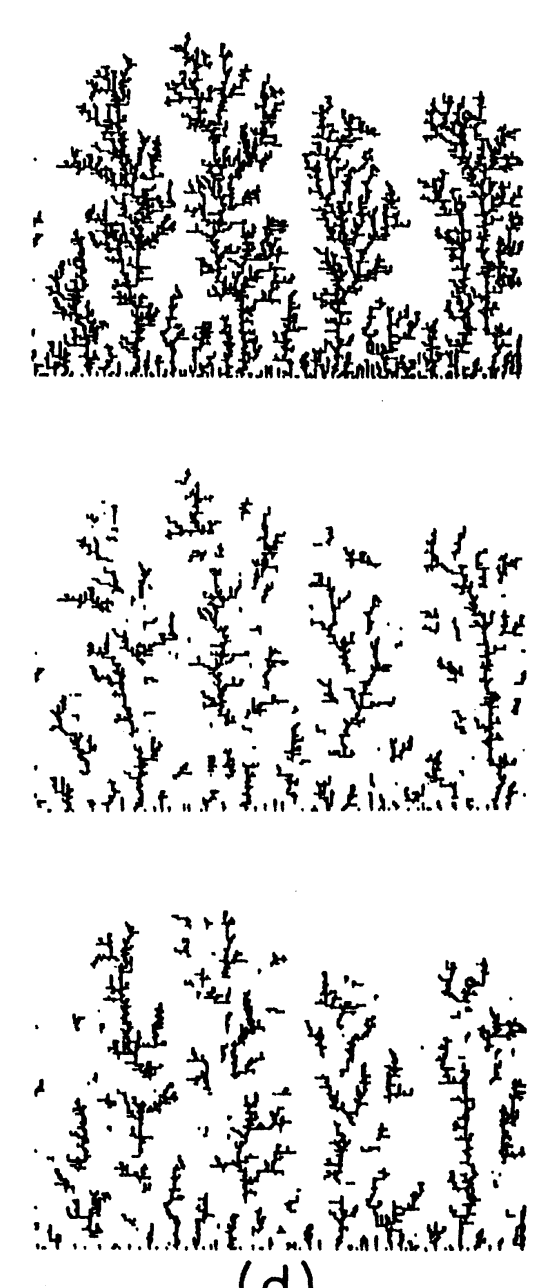

(d)
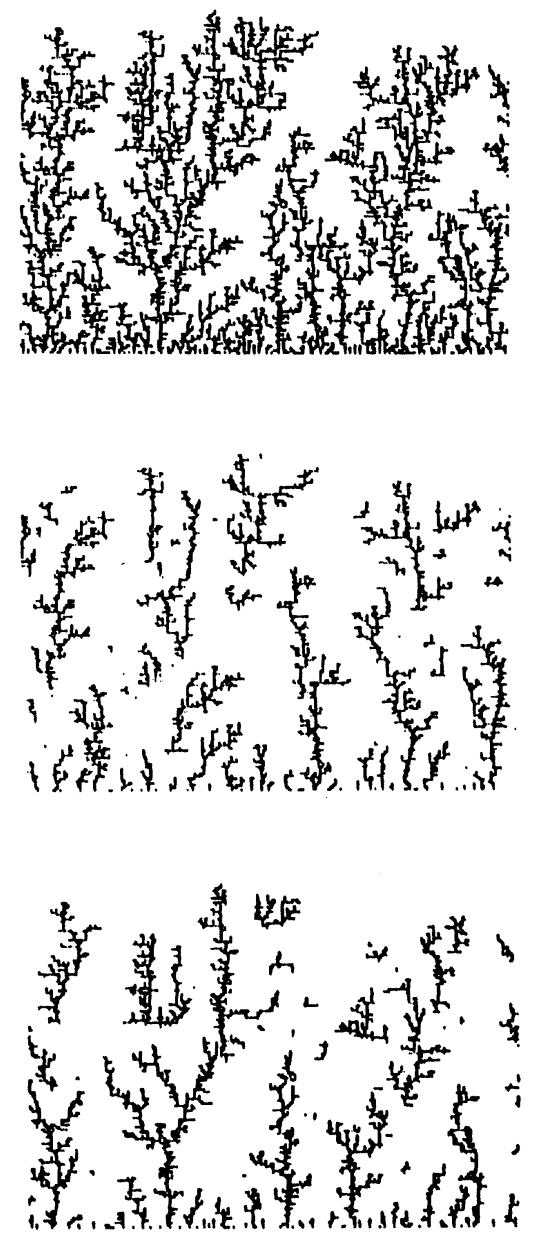

(e)

FIG. 1. (Continued).

where $P=P_{B B}$ if $B$ species lands on the surface consisting of $B$ species, $P=P_{B A}$ if $B$ species lands on the surface consisting of $A$ species. Here $\partial C / \partial n$ is the derivative normal to the interface. By introducing the sticking probability $P_{A B}\left(=P_{B A}\right)$ into the original DLA model, one can take into account the mixing effect of the two components of the DLA structure. In the following section, we consider the simulation procedure of the two Laplace equations (1a) and (1b) with the boundary conditions ( $2 a)$ and $(2 b)$.

\section{SIMULATION AND RESULT}

We consider the simulation of the aggregation process satisfying Eqs. (1a) and (1b) with the boundary conditions (2a) and (2b). The two kinds of diffusing particles can be simulated by using two different random walkers. In what follows we summarize the algorithm of the simulation process. The simulation of the two-component Laplacian growth with the boundary conditions (2a) and (2b) is carried out with the use of a simple square lattice. We consider a subset of the square lattice enclosed by a square with $200 \times 200$ (units). We start out with an occupied plate on the bottom of the square. The lateral boundary is periodic. The $A$ particle or the $B$ particle is introduced one at a time at a randomly chosen point on the upper boundary. The $A$ particle is introduced with the probability $p=C_{A} /\left(C_{A}+C_{B}\right)$ and the $B$ particle with the probability $1-p$. Each particle performs a Brownian motion. The particle continues to move until it either reaches a point adjacent to a site already occupied by a particle or until it reaches the upper boundary. When the particle reaches a point adjacent to a site already occupied by a particle and if the already occupied site is of the same kind of particles, it sticks on the aggregate with the probability $P_{A A}\left(=P_{B B}\right)$. If the already occupied site is the different kind of particles, it sticks on the aggregate with the probability $P_{A B}\left(=P_{B A}\right)$. Otherwise, the particle is reflected at the aggregate with the probability $1-P_{A A}$ or $1-P_{A B}$ and continues to move. 


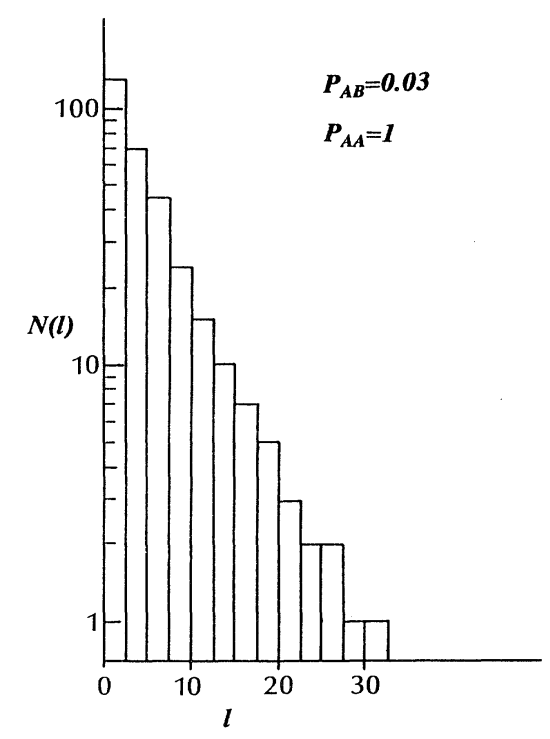

FIG. 2. Number $N(l)$ of clusters consisting of $A$ species plotted against the linear size $l$ of the cluster (in a semilogarithmic plot) for $P_{A B}=0.03, P_{A A}=1$ and $p=0.5$.

When the particle reaches the upper boundary, the random walker is annihilated. We repeat the above procedure. The deposits are grown until the height reaches 150 units. We perform the computer simulation under the condition $P_{A B}\left(=P_{B A}\right)<P_{A A}\left(=P_{B B}\right)$, assumed on what follows. The condition $P_{A B}<P_{A A}$ is selected to

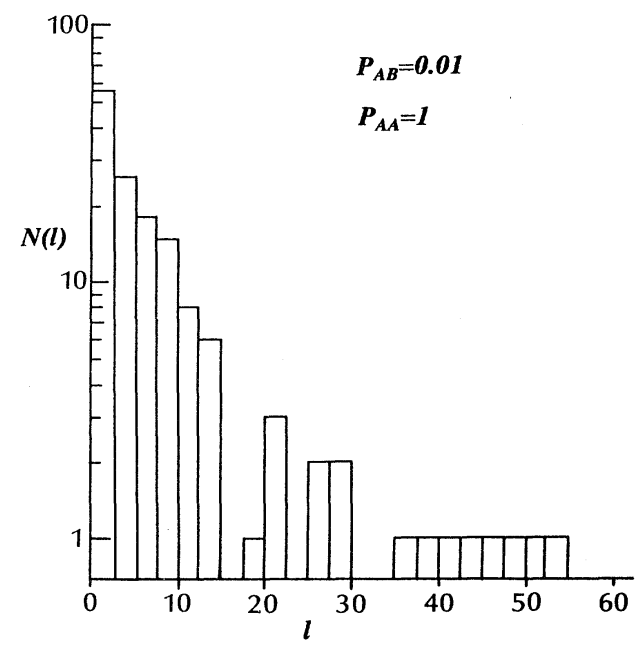

FIG. 3. Number $N(l)$ of clusters consisting of $A$ species plotted against the linear size $l$ of the cluster (in a semilogarithmic plot) for $P_{A B}=0.01, P_{A A}=1$, and $p=0.5$.

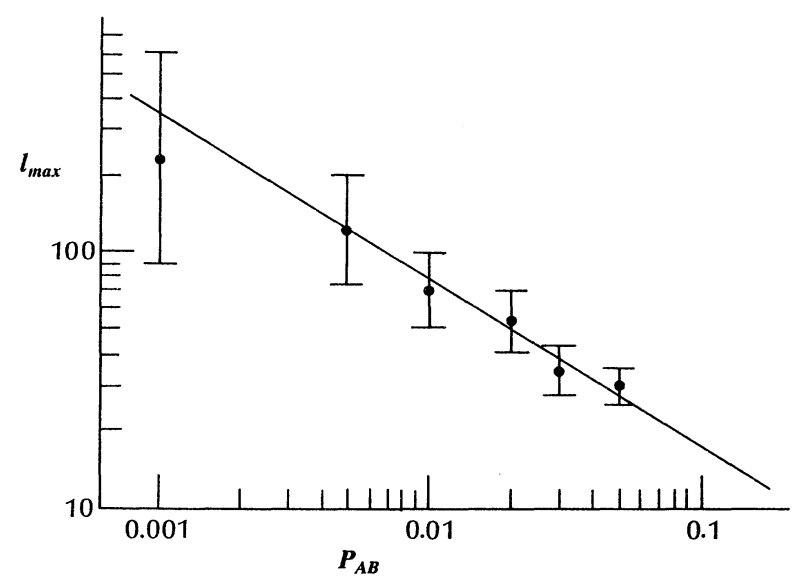

FIG. 4. The maximum size $l_{\max }$ of clusters plotted against the sticking probability $P_{A B}$ (in a log-log plot) for $P_{A A}=1$ and $p=0.5$.

study the segregation phenomena. The obvious physical meaning behind this condition is that the connection between the same species is easier than the connection between the different species. In the following, we study the three cases: (1) $P_{A A}=1$ and $p=0.5$, (2) $P_{A A}<1$ and $p=0.5$, and (3) $P_{A A}<1$ and $0<p<1$.

\section{A. The case of $P_{A A}=1$ and $p=0.5$}

Firstly, we investigate the effect of the sticking probability $P_{A B}$ between the different species on the pattern morphology. We set the sticking probability $P_{A A}$ $\left(=P_{B B}\right)$ between the same species as $P_{A A}=1$. We also consider the case of the equal concentration $p=0.5$. Figures 1 (a)-(e) show, respectively, the simulation results for $P_{A B}=0.05,0.03,0.01,0.005$, and 0.001. The upper, middle, and bottom patterns in each figure indicate, respectively, the deposits consisting of $A$ and $B$ species, only $A$ species within the composite deposit, and only $B$ species within the composite deposit. The simulation result is obtained by using the procedures outlined above. With decreasing sticking probability $P_{A B}$, the clusters consisting of the same species begin to grow. A clustering of the same species is already apparent near the value of $P_{A B}=0.01$. With a sufficiently small sticking probability $P_{A B}$, a perfect segregation occurs. The structure of the composite deposit shows the mixture consisting of the trees only with $A$ species and the trees only with $B$ species. It is found that the sticking probability $P_{A B}$ between the different species has an important effect on the pattern morphology. Figures 2 and 3 show the number $N(l)$ of the cluster consisting of the $A$ species plotted against the linear size $l$ of the cluster (in a semilogarithmic plot) for $P_{A B}=0.03$ and 0.01 . The quantity $N(l)$ in 

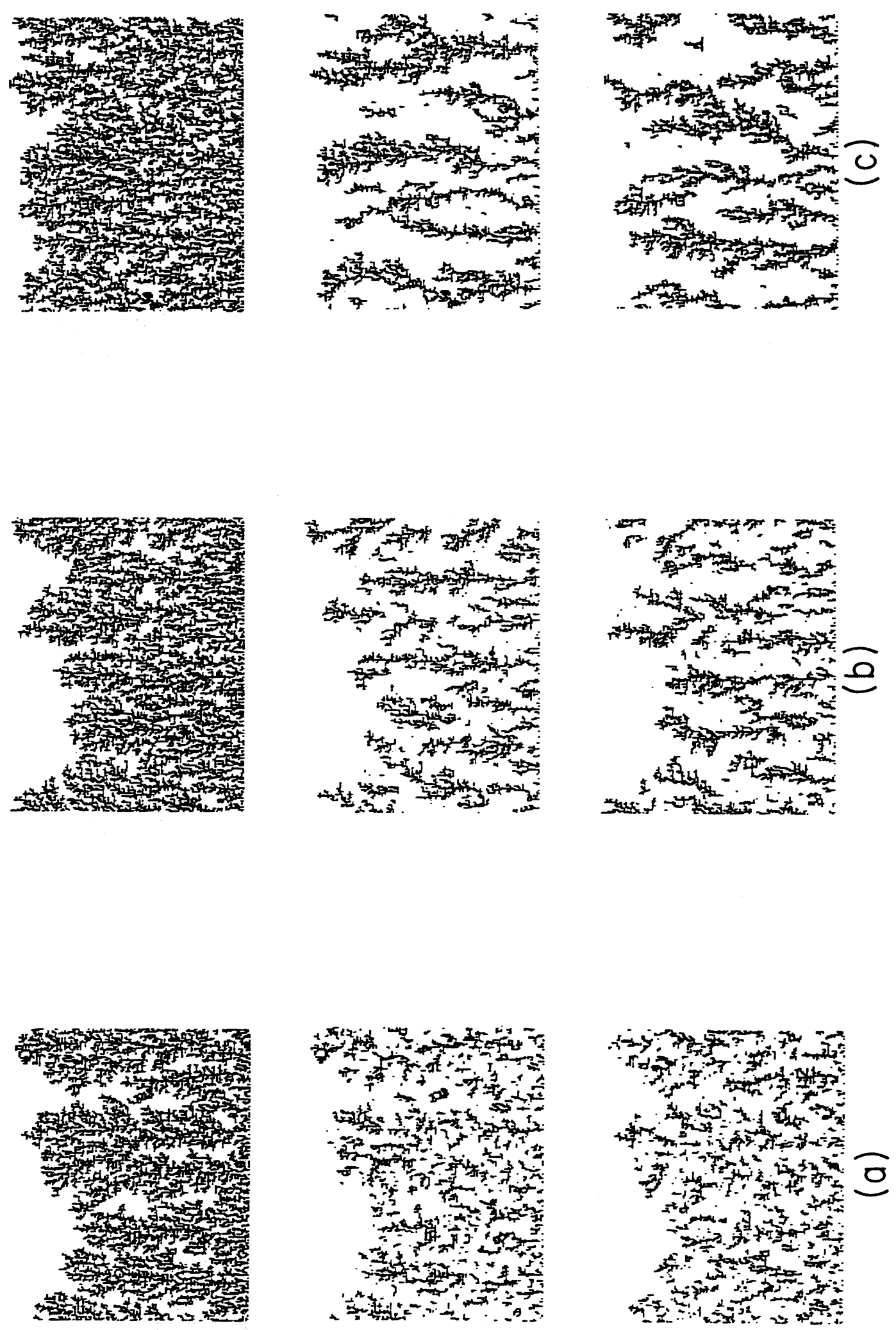

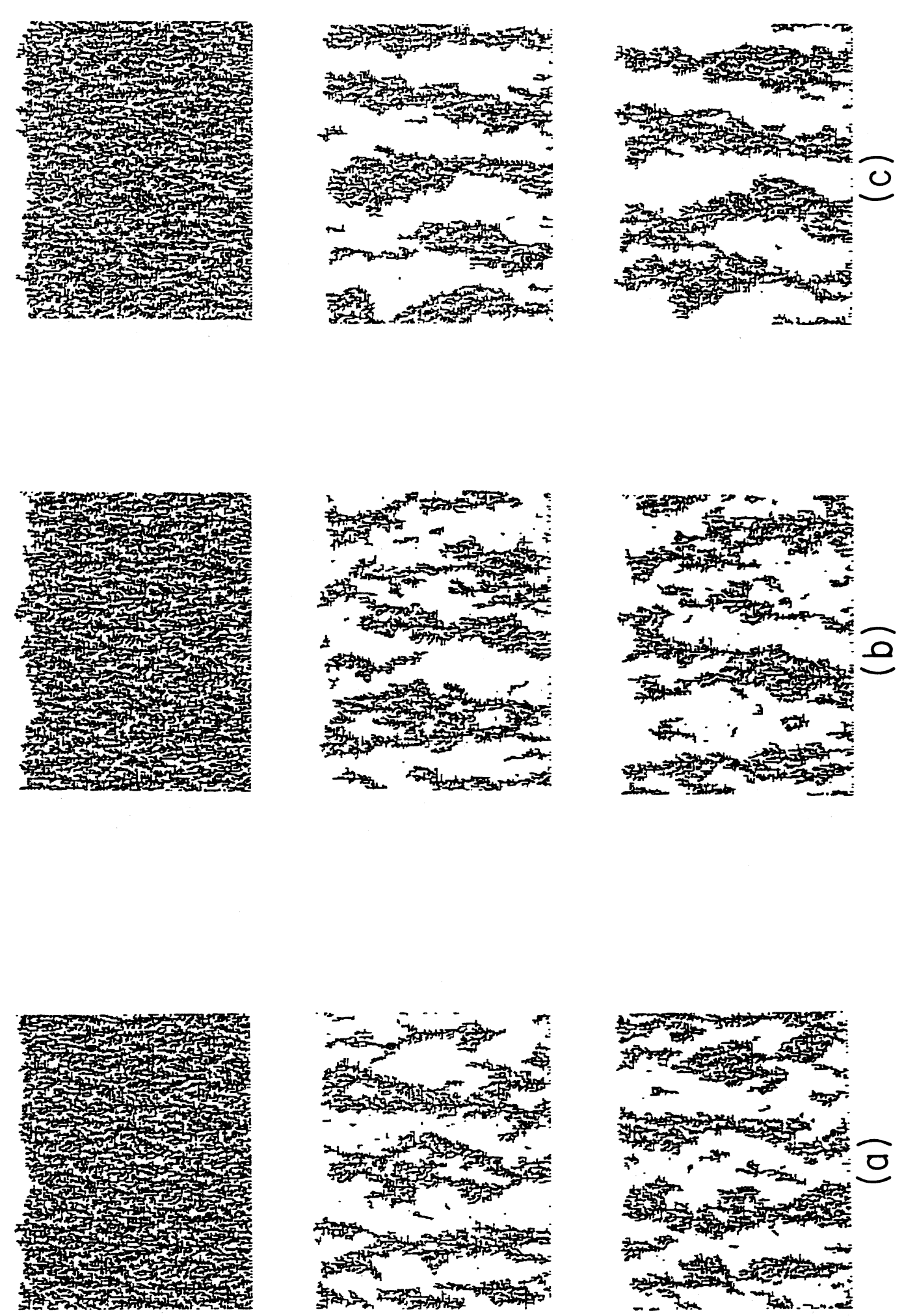

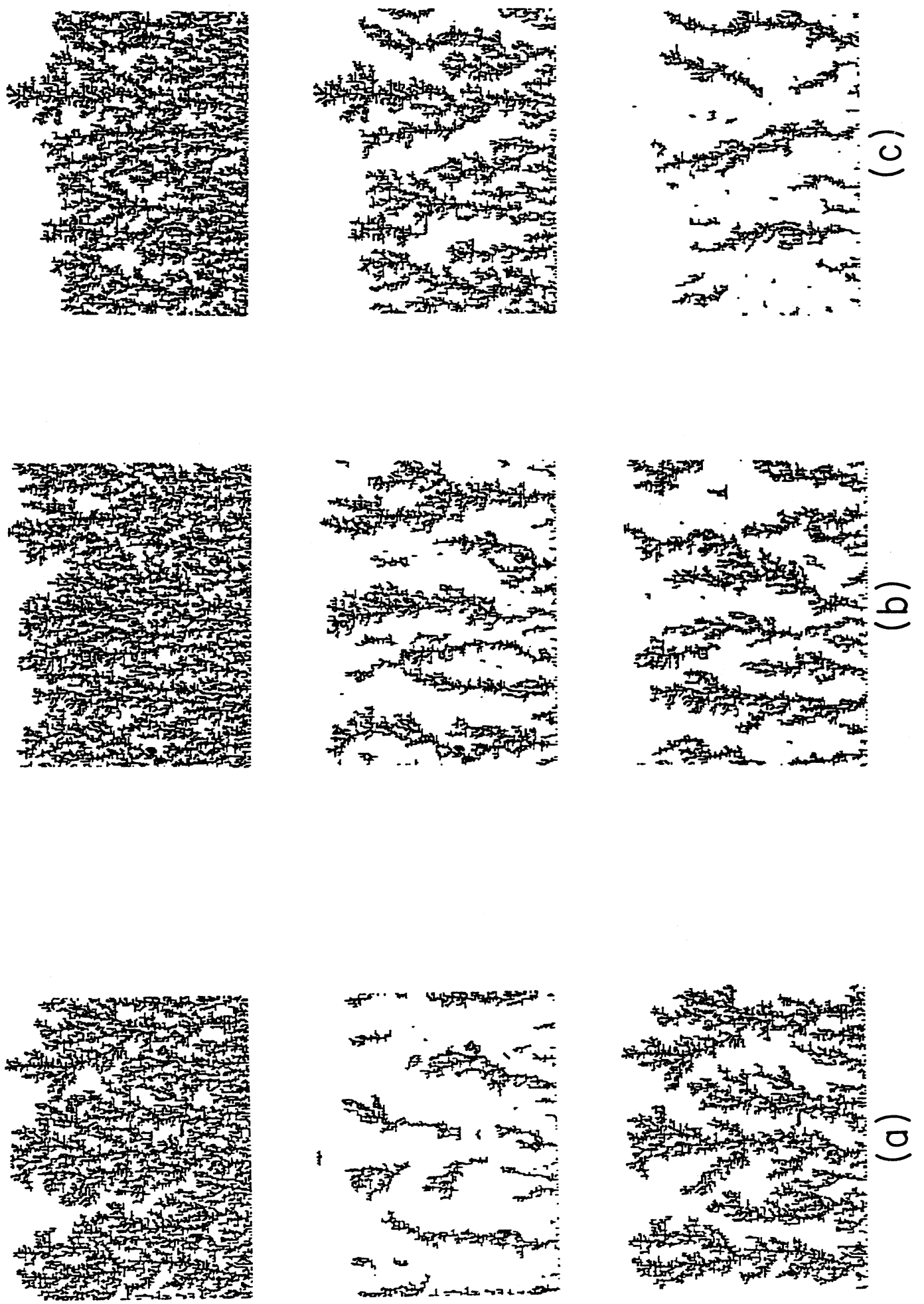

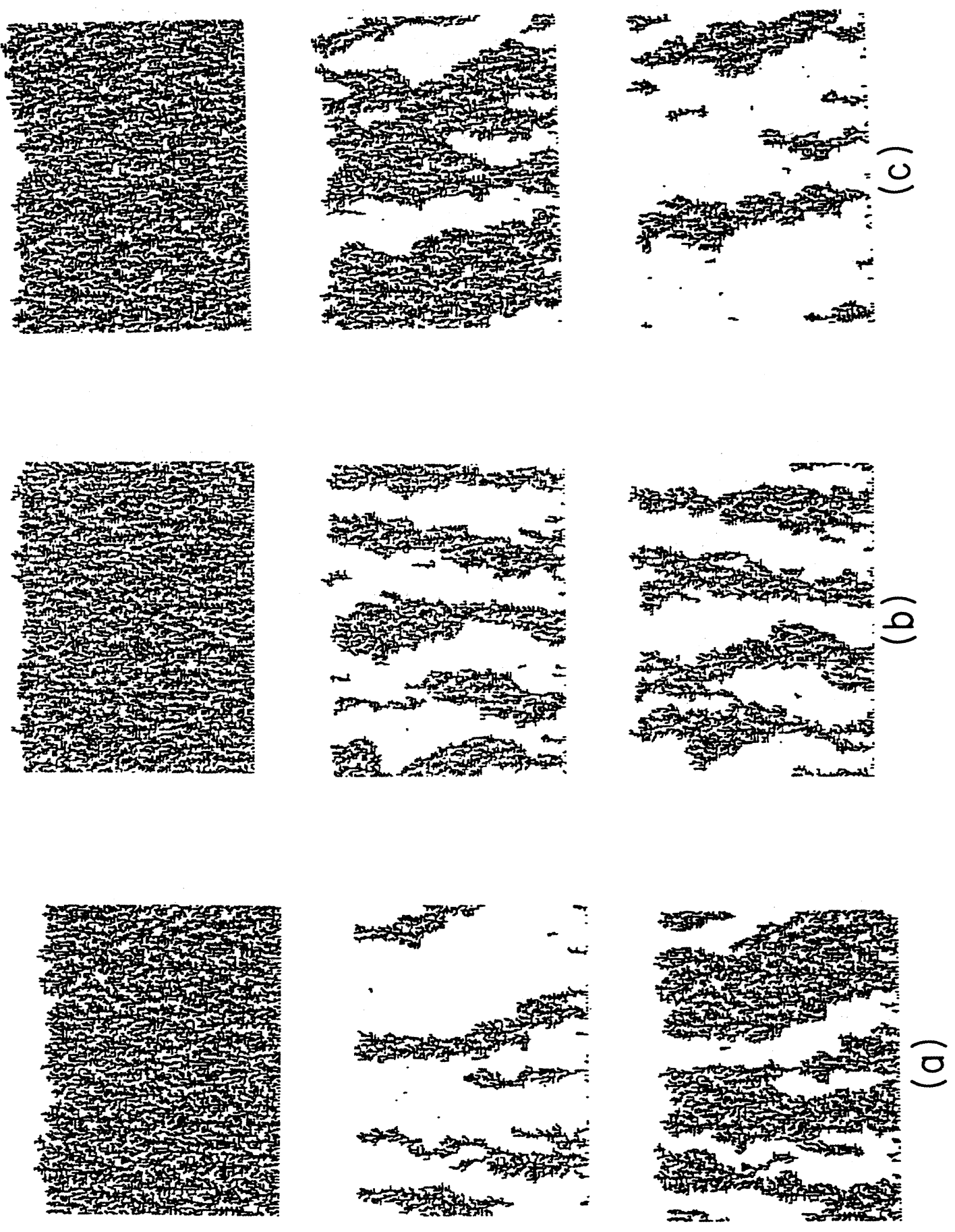
Fig. 2 shows a typical exponential decay. However, the cluster distribution in Fig. 3 does not show the exponential decay. Some large clusters appear by the clustering effect of the small sticking probability $P_{A B}$. The clustering breaks the exponential decay. Figure 4 shows the maximum size $l_{\max }$ of clusters plotted against the sticking probability $P_{A B}$ (in a log-log plot). The maximum size of clusters increases with decreasing sticking probability $P_{A B}$. The maximum size of clusters fluctuates more largely with a smaller sticking probability $P_{A B}$. The maximum size scales as

$$
l_{\max } \approx P_{A B}^{-0.64} .
$$

When the maximum size of clusters reaches to the system size $L(=200)$, the perfect segregation occurs. We have a criterion of the perfect segregation.

$$
l_{\max } \approx L \text {. }
$$

In the case of $P_{A A}=1$ and $p=0.5$, we find that the clusters of the same species grow with decreasing sticking probability $P_{A B}$ and a segregation occurs with a sufficiently small sticking probability $P_{A B}$.

$$
\text { B. The case of } P_{A A}<1 \text { and } p=0.5
$$

We consider the effect of both sticking probabilities $P_{A A}$ and $P_{A B}$ on the pattern morphology. We can obtain the simulation result by using the procedures outlined above. Figure 5 shows the simulation result for $P_{A A}=0.1$ with a variable $P_{A B}$ where $p=0.5$. The simulation results for $P_{A B}=0.05,0.01$, and 0.001 are shown, respectively, in Figs. 5(a)-5(c). With decreasing sticking probability $P_{A B}$, clusters of the same species grow. In the limit of a sufficiently small sticking probability $P_{A B}$, a perfect segregation occurs, although the individual deposits begin to show a less ramified structure than in the previous case. The overall pattern of the composite deposit becomes dense similarly to that in the single-component DLA. Figure 6 shows the simulation result for $P_{A A}=0.01$ with a variable $P_{A B}$ where $p=0.5$. The simulation results for $P_{A B}=0.08,0.005$, and 0.001 are shown, respectively, in Figs. 6(a)-6(c). With a sufficiently small $P_{A A}$, a perfect segregation occurs. The overall pattern of the composite deposit becomes more dense than in the case of $P_{A A}=0.1$. Furthermore, the segregated patterns of the composite deposit begin to show a typical striped structure. With decreasing $P_{A B}$, the striped pattern becomes more and more clear. We find that the segregated patterns of the composite deposit show the striped structure in the limit of both sufficiently small $P_{A A}$ and $P_{A B}$.

$$
\text { C. The case of } P_{A A}<1 \text { and variable } p
$$

We studied the cases of $p=0.5$ above. We here consider the effect of the concentration $p$ on the segregated pattern. By using the simulation method outlined above, we can obtain the simulation results for different values of the concentration $p$. Figures $7(\mathrm{a})-(\mathrm{c})$ show, respectively, the simulation results of $P_{A A}=0.1$ and $P_{A B}=0.001$ for $p=0.3,0.5$, and 0.7 . Figures $8(\mathrm{a})-8(\mathrm{c})$ show, respectively, the simulation results of $P_{A A}=0.01$ and $P_{A B}=0.001$ for $p=0.3,0.5$, and 0.7 . The upper, middle, and bottom patterns in each figures indicate, respectively, the composite deposits consisting of the $A$ and $B$ species, only the $A$ species within the composite deposit and only the $B$ species within the composite deposit. With increasing concentration $p$, the width of the stripes of the $A$ species becomes bigger. We find that the concentration $p$ has an important effect on the width of the stripes in the limit of both sufficiently small sticking probabilities $P_{A A}$ and $P_{A B}$.

\section{SUMMARY}

We present a diffusion-limited-aggregation (DLA) model with two components ( $A$ and $B$ species) to investigate the pattern morphology and segregation of the composite deposits. We introduce the sticking probability $P_{A B}\left(=P_{B A}\right)$ between the different species into the original DLA model. By using the computer simulation, we show that various pattern morphologies are produced with varying the sticking probabilities $P_{A B}\left(=P_{B A}\right)$ and $P_{A A}\left(=P_{B B}\right)$, where $P_{A A}\left(=P_{B B}\right)$ is the sticking probability between the same species. We analyze the segregated pattern under the condition $P_{A B}<P_{A A}$. We find that a clustering of the same species occurs with decreasing sticking probability $P_{A B}$. We show that the composite deposit becomes dense and the segregated patterns of the composite deposit show a striped structure with sufficiently small values of both sticking probabilities $P_{A B}$ and $P_{A A}$. We also show that the width of the cluster of the $A$ species increases with the concentration $p$.

\section{ACKNOWLEDGMENTS}

One of us (T.N.) acknowledges financial support received from Direccion General de Investigacion Cientifica y Tecnica (DGICYT, Spain) during the completion of this work. This research is funded by DGICYT under Project No. PB 87-0014.
[1] Kinetics of Aggregation and Gelation, edited by F. Family and D. P. Landau (North-Holland, Amsterdam, 1984).

[2] On Growth and Form, edited by H. E. Stanley and N. Ostrowsky (Nijihoff, The Hague, 1985).

[3] Fractals in Physics, edited by L. Pietronero and E. Tosatti (North-Holland, Amsterdam, 1986).

[4] H. J. Herrmann, Phys. Rep. 136, 153 (1986).

[5] P. Meakin, in Phase Transitions and Critical Phenomena, edited by C. Domb and J. L. Lebowitz (Academic, New York, 1988), Vol. 12, p. 336.

[6] R. Julien and R. Botet, Aggregation and Fractal Aggregates (World Scientific, Singapore, 1987).

[7] J. Feder, Fractals (Plenum, New York, 1988).

[8] Randon Fluctuations and Pattern Growth, edited by H. E. Stanley and N. Ostrowsky (Kluwer Academic, Dordrecht, 1988). 
[9] T. Vicsek, Fractal Growth Phenomena (World Scientific, Singapore, 1989).

[10] H. E. Stanley, A. Bunde, S. Havlin, J. Lee, E. Roman, and S. Schwarzer, Physica A 168, 23 (1990).

[11] T. A. Witten and L. M. Sander, Phys. Rev. Lett. 47, 1400 (1981); Phys. Rev. B 27, 5686 (1983).

[12] P. Meakin, Phys. Rev. A 26, 1495 (1983); 27, 2616 (1983).

[13] G. Daccord and R. Lenormand, Nature (London) 325, 41 (1987).

[14] H. Fujikawa and M. Matsushita, J. Phys. Soc. Jpn. 58, 3875 (1989).

[15] J. D. Sherwood, J. Phys. A 19, L195 (1986).

[16] M. J. King and H. Scher, Phys. Rev. A 41, 874 (1990).

[17] M. Murat and A. Aharony, Phys. Rev. Lett. 57, 1875
(1986).

[18] V. Horvath, J. Kertesz, and T. Vicsek, Europhys. Lett. 4, 1133 (1987).

[19] R. F. Voss, J. Stat. Phys. 36, 861 (1984).

[20] D. Grier, E. Ben-Jacob, Roy Clarke, and L. M. Sander, Phys. Rev. Lett. 56, 1264 (1986).

[21] T. Nagatani, Phys. Rev. A 40, 7286 (1989).

[22] J. Lee, A. Coniglio, and H. E. Stanley, Phys. Rev. A 41, 4589 (1990).

[23] T. Nagatani and H. E. Stanley, Phys. Rev. A 41, 3263 (1990).

[24] T. Nagatani, J. Lee, and H. E. Stanley, Phys. Rev. Lett. 66, 616 (1991).

[25] T. Nagatani and F. Sagués, Phys. Rev. A (to be published). 